\title{
Harmonic Evolute Surface of Tubular Surfaces via B-Darboux Frame in Euclidean 3-Space
}

\author{
E. M. Solouma $\mathbb{D}^{1,2}$ and Ibrahim AL-Dayel $\mathbb{D}^{1}$ \\ ${ }^{1}$ Department of Mathematics and Statistics, College of Science, Imam Mohammad Ibn Saud Islamic University, Saudi Arabia \\ ${ }^{2}$ Department of Mathematics and Computer Science, Faculty of Science, Beni-Suef University, Egypt
}

Correspondence should be addressed to E. M. Solouma; emmahmoud@imamu.edu.sa

Received 21 August 2021; Revised 19 September 2021; Accepted 22 September 2021; Published 18 November 2021

Academic Editor: Manuel De Le n

Copyright (C) 2021 E. M. Solouma and Ibrahim AL-Dayel. This is an open access article distributed under the Creative Commons Attribution License, which permits unrestricted use, distribution, and reproduction in any medium, provided the original work is properly cited.

\begin{abstract}
In this article, we look at a surface associated with real-valued functions. The surface is known as a harmonic surface, and its unit normal vector and mean curvature have been used to characterize it. We use the Bishop-Darboux frame ( $\mathbb{B}$-Darboux frame) in Euclidean 3-space $\mathrm{E}^{3}$ to study and explain the geometric characteristics of the harmonic evolute surfaces of tubular surfaces. The characterizations of the harmonic evolute surface's $\varrho$ and $\varsigma$ parameter curves are evaluated, and then, they are compared. Finally, an example of a tubular surface's harmonic evolute surface is presented, along with visuals of these surfaces.
\end{abstract}

\section{Introduction}

Darboux frame is a differential geometric approach for evaluating curves and surfaces. The Frenet frame is the most well-known frame field, although there are others, such as the Darboux frame. There have been several instances of frame studies of this sort, for example, see $[1,2]$.

The Bishop frame is a way for defining a moving frame that is well defined despite due to the vanished of curve's second derivative [3]. Parallel transferring each element of an orthogonal frame along a curve is as easy as parallel transferring each element of the frame.

In $\mathrm{E}^{3}$, the geometrical position of the points at the inverse distance in terms of multiplication of the mean curvature from the surface is known as the harmonic evolute surface of a tubular surface. The harmonic evolute surface can be defined for a nonminimal surface.

Let $\mathbb{M}: \Omega(\rho, \varsigma)$ be a surface associated with real-valued functions, $\mathbb{Q}(\rho, \varsigma)$ and $H(\rho, \varsigma)$ which, respectively, are the normal vector and mean curvature of $\Omega$. The harmonic surface $\Gamma(\rho, \varsigma)$ has a parameterized description as follows:

$$
\Gamma(\rho, \varsigma)=\Omega(\rho, \varsigma)+\frac{1}{H(\rho, \varsigma)} \mathbb{Q}(\rho, \varsigma)
$$

Many researches on harmonic evolute surfaces have been published, some of which may be included here (see [4-7]). The geometric features of the harmonic evolute surface of a tubular surface via $\mathbb{B}$-Darboux frame have inspired us to study the geometric characteristics of the harmonic evolute surface of a tubular surface. As a result, the tubular surface and the harmonic evolute surface generated from this surface will be compared and interpreted.

\section{Preliminaries}

Consider the Euclidean 3-space $\mathrm{E}^{3}$. It contains the metric as follows:

$$
\langle,\rangle=d \varepsilon_{1}^{2}+d \varepsilon_{2}^{2}+d \varepsilon_{3}^{2},
$$

where $\left(\varepsilon_{1}, \varepsilon_{2}, \varepsilon_{3}\right) \in \mathrm{E}^{3}$ 's coordinate system. 
For a regular curve $\mu(\rho)$ lying on surface $\mathbb{M}=\Omega(\rho, \varsigma)$, we denote the Darboux frame on the surface by $\{T, P, \mathbb{Q}\}$, where $P=\mathbb{Q} \times T$ and $\mathbb{Q}$ is just surface's normal $[1,8]$. Then,

$$
\left[\begin{array}{l}
T(\rho) \\
P(\rho) \\
\mathbb{Q}(\rho)
\end{array}\right]_{\rho}=\left[\begin{array}{ccc}
0 & \kappa_{g}(\rho) & \kappa_{n}(\rho) \\
-\kappa_{g}(\rho) & 0 & \tau_{g}(\rho) \\
-\kappa_{n}(\rho) & -\tau_{g}(\rho) & 0
\end{array}\right]\left[\begin{array}{c}
T(\rho) \\
P(\rho) \\
\mathbb{Q}(\rho)
\end{array}\right],
$$

where even the geodesic curvature $\kappa_{g}$, normal curvature $\kappa_{n}$, and relative torsion $\tau_{g}$ are defined as:

$$
\tau_{g}=\left\langle P^{\prime}, \mathbb{Q}\right\rangle, \kappa_{n}=\left\langle T^{\prime}, \mathbb{Q}\right\rangle, \kappa_{g}=\left\langle T^{\prime}, P\right\rangle
$$

In matrix form, the $\mathbb{B}$-Darboux frame's variation equation $\left\{T, \mathbb{B}_{1}, \mathbb{B}_{2}\right\}$ on the surface $\mathbb{M}$ is as shown below [1]:

$$
\left[\begin{array}{c}
T(\rho) \\
\mathbb{B}_{1}(\rho) \\
\mathbb{B}_{2}(\rho)
\end{array}\right]_{\rho}=\left[\begin{array}{ccc}
0 & \zeta_{1}(\rho) & \zeta_{2}(\rho) \\
-\zeta_{1}(\rho) & 0 & 0 \\
-\zeta_{2}(\rho) & 0 & 0
\end{array}\right]\left[\begin{array}{c}
T(\rho) \\
\mathbb{B}_{1}(\rho) \\
\mathbb{B}_{2}(\rho)
\end{array}\right],
$$

where $\zeta_{1}$ and $\zeta_{2}$, the $\mathbb{B}$-Darboux curvatures, are acquired in the following way:

$$
\begin{aligned}
& \zeta_{1}=\kappa_{g} \sin \phi+\kappa_{n} \cos \phi, \\
& \zeta_{2}=\kappa_{n} \sin \phi-\kappa_{g} \cos \phi .
\end{aligned}
$$

Also, the relation matrix given by

$$
\left[\begin{array}{c}
T(\rho) \\
\mathbb{B}_{1}(\rho) \\
\mathbb{B}_{2}(\rho)
\end{array}\right]=\left[\begin{array}{ccc}
1 & 0 & 0 \\
0 & \sin \phi & \cos \phi \\
0 & -\cos \phi & \sin \phi
\end{array}\right]\left[\begin{array}{c}
T(\rho) \\
P(\rho) \\
\mathbb{Q}(\rho)
\end{array}\right],
$$

such that angle $\phi$ between $\mathbb{Q}$ and $\mathbb{B}_{1}$ is acquired around

$$
\phi-\phi_{0}=\int \tau_{g} d t
$$

for any arbitrary constant $\phi_{0}$. The relation among $\mathbb{B}$-Darboux's curvatures and Darboux's curvatures satisfies

$$
\zeta_{1}^{2}+\zeta_{2}^{2}=\kappa_{g}^{2}+\kappa_{n}^{2}
$$

Let $\mathbb{M}: \Omega(\rho, \varsigma)$ be regular surface in $\mathrm{E}^{3}$, then the $\Omega$ 's unit normal vector $\mathbb{Q}$ can be written as

$$
\mathbb{Q}=\frac{\Omega_{\rho} \times \Omega_{\varsigma}}{\left\|\Omega_{\rho} \times \Omega_{\varsigma}\right\|},
$$

where $\Omega_{\rho}=\partial \Omega / \partial \rho$ and $\Omega_{\varsigma}=\partial \Omega / \partial \varsigma$. The Gaussian curvature $K$ and mean $H$ curvature were also provided by [9-11]

$$
\begin{aligned}
& K=\frac{h_{11} h_{22}-h_{12}^{2}}{g_{11} g_{22}-g_{12}^{2}}, \\
& H=\frac{g_{11} h_{22}+g_{22} h_{11}-2 g_{12} h_{12}}{2\left(g_{11} g_{22}-g_{12}^{2}\right)},
\end{aligned}
$$

where $g_{11}=\left\|\Omega_{\rho}\right\|^{2}, g_{12}=\left\langle\Omega_{\rho}, \Omega_{\zeta}\right\rangle, g_{22}=\left\|\Omega_{\varsigma}\right\|^{2}, h_{11}=\left\langle\Omega_{\rho \rho}\right.$, $\mathbb{Q}\rangle, h_{12}=\left\langle\Omega_{\rho \varsigma}, \mathbb{Q}\right\rangle$, and $h_{22}=\left\langle\Omega_{\varsigma \varsigma}, \mathbb{Q}\right\rangle$.

\section{Obtaining Tubular Surface via $\mathbb{B}$-Darboux Frame}

Let $\mu(\rho)$ be an arc-length-parameterized curve in $\mathrm{E}^{3}$. Then, the tubular surface via the $\mathbb{B}$-Darboux frame has the parametrization $[2,12,13]$ :

$$
\Omega(\rho, \varsigma)=\mu(\rho)+r\left[\cos \varsigma \mathbb{B}_{1}(\rho)+\sin \varsigma \mathbb{B}_{2}(\rho)\right]
$$

where $r$ is constant and sphere's radius. The velocity vectors of $\Omega$ along $\mu$ are

$$
\begin{aligned}
& \Omega_{\rho}=[1-r \lambda(\rho, \varsigma)] T(\rho), \\
& \Omega_{\varsigma}=-r \sin \varsigma \mathbb{B}_{1}(\rho)+r \cos \varsigma \mathbb{B}_{2}(\rho),
\end{aligned}
$$

where $\lambda(\rho, \varsigma)=\kappa_{g}(\rho) \sin (\phi-\varsigma)+\kappa_{n}(\rho) \cos (\phi-\varsigma)$. As a result, the trying to follow are the features of $\Omega$ 's first fundamental form:

$$
g_{11}=(1-r \lambda)^{2}, g_{12}=0, g_{22}=r^{2}
$$

The $\Omega$ 's unit surface normal vector $\mathbb{Q}_{\Omega}$, from the other hand, is acquired by

$$
\mathbb{Q}_{\Omega}=-\cos \varsigma \mathbb{B}_{1}(\rho)-\sin \varsigma \mathbb{B}_{2}(\rho) .
$$

$\Omega$ 's second order partial diffrentials are discovered as

$$
\begin{aligned}
& \Omega_{\rho \rho}=\left(-r \lambda_{\rho}\right) T(\rho)+\zeta_{1}(1-r \lambda) \mathbb{B}_{1}(\rho)+\zeta_{2}(1-r \lambda) \mathbb{B}_{2}(\rho), \\
& \Omega_{\rho \varsigma}=\left(-r \lambda_{\varsigma}\right) T(\rho), \\
& \Omega_{\varsigma \varsigma}=-r \cos \varsigma \mathbb{B}_{1}(\rho)-r \sin \varsigma \mathbb{B}_{2}(\rho) .
\end{aligned}
$$

The coefficients of the second fundamental form are derived using (13) as illustrated below.

$$
h_{11}=-\lambda(1-r \lambda), h_{12}=0, h_{22}=r .
$$

Thus, the Gaussian curvature $K_{\Omega}$ and mean curvature $H_{\Omega}$ functions are calculated as

$$
K_{\Omega}(\rho, \varsigma)=-\frac{\lambda}{r(1-r \lambda)}, H_{\Omega}(\rho, \varsigma)=\frac{1-2 r \lambda}{2 r(1-r \lambda)} .
$$

Theorem 1. The tubular surface $M: \Omega(\mathrm{Q}, \varsigma)$ via the $\mathbb{B}$-Darboux frame described by (12) is developable iff 


$$
\tan (\phi-\varsigma)=-\frac{\kappa_{n}(\rho)}{\kappa_{g}(\rho)}
$$

Theorem 2. The tubular surface $M: \Omega(\varrho, \varsigma)$ via the $\mathbb{B}$-Darboux frame described by (12) is minimal iff the following equation satisfies

$$
1-2 r\left[\kappa_{g}(\rho) \sin (\phi-\varsigma)+\kappa_{n}(\rho) \cos (\phi-\varsigma)\right]=0
$$

Corollary 3. Let $\mathbb{M}: \Omega(\varrho, \varsigma)$ be tubular surface via the $\mathbb{B}$-Darboux frame described by (12). The $\mathrm{Q}$-parameter is then not geodesic curves but $\varsigma$-parameter is geodesic curves.

Proof. Let $\Omega$ be a tubular surface defined by Equation (12), and we get process and techniques from Equations (15) and (16)

$$
\mathbb{Q}_{\Omega} \times \Omega_{\rho \rho} \neq 0 \text { and } \mathbb{Q}_{\Omega} \times \Omega_{\varsigma \varsigma}=0
$$

where $\times$ stands for cross product. So, the proof is clear in such scenario.

Corollary 4. Let $M: \Omega(\varrho, \varsigma)$ be tubular surface via the $\mathbb{B}$-Darboux frame described by (12). The s-parameter is not asymptotic curves but @-parameter is then asymptotic curves iff

$$
\kappa g(\varrho)=\frac{1}{r \sin (\phi-\varsigma)}
$$

Proof. If $\Omega$ is a tubular surface as defined by Equation (12), from Equations (15) and (16), we have $\left\langle\mathbb{Q}_{\Omega}, \Omega_{\rho \rho}\right\rangle$ $=\zeta_{1}(1-r \lambda)=0$ if only and only if $\lambda=1 / r$ or equivalently $\kappa_{g}(\rho)=1 /(r \sin (\phi-\varsigma))$. But $\left\langle\mathbb{Q}_{\Omega}, \Omega_{\varsigma \varsigma}\right\rangle=r \neq 0$, which completes the proof.

Corollary 5. Let $M: \Omega(\varrho, \varsigma)$ be tubular surface via the $\mathbb{B}$-Darboux frame described by (12). The $\mathrm{Q}$ and $\mathrm{s}$-parameters are then principal curves.

Proof. Let $\Omega$ be a tubular surface defined by Equation (12), and we get process and techniques from Equations (14) and (17), then we have

$$
g_{12}=h_{12}=0
$$

Then, the proof is clear.

Corollary 6. The tubular surface $M: \Omega(\mathrm{Q}, \varsigma)$ via the $\mathbb{B}$-Darboux frame described by (12) is a $(K \Omega, H \Omega)$-Weingarten surface.

Proof. If the Jacobi equation $\left(K_{\Omega}, H_{\Omega}\right)=0$ occurs between the Gaussian curvature $K_{\Omega}$ and the mean curvature $H_{\Omega}$ on a surface, it is termed a Weingarten surface (see [10]). Now, if $\Omega$ be a tubular surface defined by Equation (12) and from Equation (18), we get

$$
\left(\begin{array}{c}
\left(K_{\Omega}\right)_{\rho}=-\frac{\lambda_{\rho}}{r(1-r \lambda)^{2}},\left(K_{\Omega}\right)_{\varsigma}=-\frac{\lambda_{\varsigma}}{r(1-r \lambda)^{2}}, \\
\left(H_{\Omega}\right)_{\rho}=-\frac{\lambda_{\rho}}{2(1-r \lambda)^{2}},\left(H_{\Omega}\right)_{\varsigma}=-\frac{\lambda_{\varsigma}}{2(1-r \lambda)^{2}} .
\end{array}\right.
$$

It is clear that $\left(H_{\Omega}\right)_{\rho}\left(K_{\Omega}\right)_{\zeta}=\left(H_{\Omega}\right)_{\zeta}\left(K_{\Omega}\right)_{\rho}$.

Corollary 7. The tubular surface $M: \Omega(\varrho, \varsigma)$ via the $\mathbb{B}$-Darboux frame defined by (12) is a $(K \Omega, H \Omega)$-linear Weingarten surface iff

$$
\lambda=\frac{2 r c+b}{2\left(a+r b-r^{2} c\right)},
$$

where $c, c_{1}$, and $c_{2}$ are not all zero real numbers.

Proof. A surface $\Omega$ is said to be a $\left(K_{\Omega}, H_{\Omega}\right)$-linear Weingarten surface if the curvatures $K_{\Omega}$ and $H_{\Omega}$ of $\Omega$ satisfy $a K_{\Omega}+b$ $H_{\Omega}=c$, where $a, b, c \in R$ (see [10]). Then, one can see that

$$
\lambda=\frac{2 r c+b}{2\left(a+r b-r^{2} c\right)},
$$

where $a, b$, and $c$ are not all zero real numbers.

\section{Constructing the Harmonic Surface of Tubular Surface via $\mathbb{B}$-Darboux Frame}

We now concentrate on the parametrization of $\mathbb{M}^{*}$ harmonic surface of $\mathbb{M}$ by using (12), (15), and (18). We define $\mathbb{M}^{*}$ as follows:

$$
\Gamma(\mathrm{\varrho}, \varsigma)=\mu(\mathrm{\varrho})+\rho(\mathrm{\varrho}, \varsigma)\left[\cos \varsigma \mathbb{B}_{1}(\mathrm{\varrho})+\sin \varsigma \mathbb{B}_{2}(\mathrm{\varrho})\right]
$$

where $\rho(\rho, \varsigma)=-(r /(1-2 r \lambda(\rho, \varsigma)))$. The $\Gamma^{\prime}$ 's velocity vectors are

$$
\begin{aligned}
& \Gamma_{\rho}=(1-\lambda \rho) T(\rho)+\rho_{\rho} \cos \varsigma \mathbb{B}_{1}(\rho)+\rho_{\rho} \sin \varsigma \mathbb{B}_{2}(\rho), \\
& \Gamma_{\varsigma}=\left[\rho_{\zeta} \cos \varsigma-\rho \sin \varsigma\right] \mathbb{B}_{1}(\rho)+\left[\rho_{\zeta} \sin \varsigma+\rho \cos \varsigma\right] \mathbb{B}_{2}(\rho) .
\end{aligned}
$$

As a result, the features of $\Gamma$ 's first fundamental forms

$$
g_{11}^{*}=\rho_{\rho}^{2}+(1-\lambda \rho)^{2}, g_{12}^{*}=\rho_{\rho} \rho_{\varsigma}, g_{22}^{*}=\rho^{2}+\rho_{\varsigma}^{2} \text {. }
$$

The $\Gamma$ 's unit normal vector $\mathbb{Q}_{\Gamma}$, from the other hand, is acquired by 


$$
\begin{aligned}
\mathbb{Q}_{\Gamma}= & \frac{1}{\sqrt{\rho^{2} \rho_{\rho}^{2}+\left(\rho^{2}+\rho_{\varsigma}^{2}\right)(1-\lambda \rho)^{2}}} \\
& \cdot\left\{\rho \rho_{\rho} T(\rho)-(1-\lambda \rho)\left(\rho_{\varsigma} \sin \varsigma+\rho \cos \varsigma\right) \mathbb{B}_{1}(\rho)\right. \\
& \left.+(1-\lambda \rho)\left(\rho_{\varsigma} \cos \varsigma-\rho \sin \varsigma\right) \mathbb{B}_{2}(\rho)\right\} .
\end{aligned}
$$

$\Gamma$ 's second-order partial differentials are discovered as

$$
\begin{aligned}
\Gamma_{\rho \rho}= & -\left[2 \lambda \rho_{\rho}+\lambda_{\rho} \rho\right] T+\left[\zeta_{1}(1-\lambda \rho)+\rho_{\rho \rho} \cos \varsigma\right] \mathbb{B}_{1} \\
& +\left[\zeta_{2}(1-\lambda \rho)+\rho_{\rho \rho} \sin \varsigma\right] \mathbb{B}_{2}, \\
\Gamma_{\rho \varsigma}= & -(\lambda \rho)_{\varsigma} T+\left[\rho_{\rho \varsigma} \cos \varsigma-\rho_{\rho} \sin \varsigma\right] \mathbb{B}_{1} \\
& +\left[\rho_{\rho \varsigma} \sin \varsigma+\rho_{\rho} \cos \varsigma\right] \mathbb{B}_{2}, \\
\Gamma_{\zeta \varsigma}= & {\left[\left(\rho_{\varsigma \varsigma}-\rho\right) \cos \varsigma-2 \rho_{\varsigma} \sin \varsigma\right] \mathbb{B}_{1} } \\
& +\left[\left(\rho_{\varsigma \varsigma}-\rho\right) \sin \varsigma+2 \rho_{\varsigma} \cos \varsigma\right] \mathbb{B}_{2} .
\end{aligned}
$$

The second fundamental form coefficients are computed using (29) and (30) as follows:

$$
\begin{aligned}
h_{11}^{*}= & \frac{1}{\sqrt{\rho^{2} \rho_{\rho}^{2}+\left(\rho^{2}+\rho_{\varsigma}^{2}\right)(1-\lambda \rho)^{2}}} \\
& \cdot\left\{(1-\lambda \rho)\left[(1-\lambda \rho)\left[\rho_{\varsigma}\left(\zeta_{2} \cos \varsigma-\zeta_{1} \sin \varsigma\right)-\lambda \rho\right]-\rho \rho_{\rho \rho}\right]\right. \\
& \left.-\rho \rho_{\rho}\left(2 \lambda \rho_{\rho}+\lambda_{\rho} \rho\right)\right\}, \\
h_{12}^{*}= & \frac{(1-\lambda \rho)\left(\rho_{\rho} \rho_{\varsigma}-\rho \rho_{\rho \varsigma}\right)-\rho \rho_{\rho}(\lambda \rho)_{\varsigma}}{\sqrt{\rho^{2} \rho_{\rho}^{2}+\left(\rho^{2}+\rho_{\zeta}^{2}\right)(1-\lambda \rho)^{2}}}, \\
h_{22}^{*}= & \frac{(1-\lambda \rho)\left[2 \rho_{\varsigma}^{2}-\rho\left(\rho_{\varsigma \varsigma}-\rho\right)\right]}{\sqrt{\rho^{2} \rho_{\rho}^{2}+\left(\rho^{2}+\rho_{\varsigma}^{2}\right)(1-\lambda \rho)^{2}}} .
\end{aligned}
$$

Thus, the Gaussian curvature $K_{\Gamma}$ and mean curvature $H_{\Gamma}$ functions are calculated as

$$
\begin{aligned}
& \left\{(1-\lambda \rho)\left[(1-\lambda \rho)\left[\rho_{\varsigma}\left(\zeta_{2} \cos \varsigma-\zeta_{1} \sin \varsigma\right)-\lambda \rho\right]-\rho \rho_{\rho \rho}\right]-\rho \rho_{\rho}\left(2 \lambda \rho_{\rho}+\lambda_{\rho} \rho\right)\right\} \\
& K_{\Gamma}=\frac{\left\{(1-\lambda \rho)\left[2 \rho_{\varsigma}^{2}-\rho\left(\rho_{\varsigma \varsigma}-\rho\right)\right]\right\}-\left\{(1-\lambda \rho)\left(\rho_{\rho} \rho_{\varsigma}-\rho \rho_{\rho \varsigma}\right)-\rho \rho_{\rho}(\lambda \rho)_{\varsigma}\right\}^{2}}{\left[\rho^{2} \rho_{\rho}^{2}+\left(\rho^{2}+\rho_{\varsigma}^{2}\right)(1-\lambda \rho)^{2}\right]^{2}}, \\
& H_{\Gamma}=\frac{\left.\left[\rho_{\rho}^{2}+(1-\lambda \rho)^{2}\right]\left\{(1-\lambda \rho)\left[2 \rho_{\varsigma}^{2}-\rho\left(\rho_{\varsigma \varsigma}-\rho\right)\right]\right\}+\left[\rho^{2}+\rho_{\varsigma}^{2}\right]\left\{(1-\lambda \rho)\left[(1-\lambda \rho)\left[\rho_{\varsigma}\left(\zeta_{\zeta} \cos \varsigma-\zeta_{1} \sin \varsigma\right)-\lambda \rho\right]-\rho \rho_{\rho \rho}\right]-\rho \rho_{\rho}\left(2 \lambda \rho_{\rho}+\lambda_{\rho} \rho\right)\right\}-2 \rho_{\rho} \rho_{\varsigma}\left\{(1-\lambda \rho)\left(\rho_{\rho} \rho_{\varsigma}-\rho \rho_{\rho \varsigma}\right)-\rho \rho_{\rho}(\lambda \rho)\right)_{\varsigma}\right\}}{2\left[\rho^{2} \rho_{\rho}^{2}+\left(\rho^{2}+\rho_{\varsigma}^{2}\right)(1-\lambda \rho)^{2}\right]^{3 / 2}} .
\end{aligned}
$$

Theorem 8. The harmonic evolute surface $\mathbb{M}^{*}$ defined by (27) of tubular surface (12) via $\mathbb{B}$-Darboux frame is neither flat nor minimal.

Corollary 9. Let $\mathbb{M}^{*}$ be harmonic evolute surface (27) of tubular surface (12) via $\mathbb{B}$-Darboux frame in $E^{3}$. The $\mathrm{Q}$ and $\varsigma$-parameters are then principal curves iff $\rho=$ constant.

Proof. If and only if $g_{12}^{*}$ and $h_{12}^{*}$, the coefficients of the first and second fundamental forms, respectively, vanish, the parameter curves of $\mathbb{M}^{*}$ are lines of curvature. So, $g_{12}^{*}=h_{12}^{*}=0$ if $\rho$ is a nonzero constant, according to (29) and (32). As a result, the evidence is complete.

Corollary 10. Let $\mathbb{M}^{*}$ be harmonic evolute surface (27) of tubular surface (12) via $\mathbb{B}$-Darboux frame in $E^{3}$. Then, the following are satisfying.

(1) $\mathbb{M}^{*}$ 's $\mathrm{Q}$-parameter curves not possible asymptotic curves
(2) $\mathbb{M}^{*}$ 's $\varsigma$-parameter curves are asymptotic curves iff $\rho$ satisfies the 2nd-order differential equation

$$
\rho \rho_{\varsigma \varsigma}-2 \rho_{\varsigma}^{2}-\rho^{2}=0
$$

Proof. If the normal curvature of the parameter curves is zero everywhere, they are called asymptotic curves on the surface. If this is the case, from (30) and (32), we have

$$
\text { (1) } \begin{aligned}
h_{11}^{*} & =\left\langle\Gamma_{\rho \rho}, \mathbb{Q}_{\Gamma}\right\rangle=-(1-\lambda \rho)\left[\lambda(1-\lambda \rho)+\rho_{\rho \rho}\right]+\rho_{\rho}(2 \lambda \\
& \left.\rho_{\rho}+\lambda_{\rho} \rho\right) / \sqrt{\rho_{\rho}^{2}+(1-\lambda \rho)^{2}} \neq 0,
\end{aligned}
$$

which means that $\rho$-parameter curves are not asymptotic curves.

(2) $h_{22}^{*}=\left\langle\Gamma_{\varsigma \varsigma}, \mathbb{Q}_{\Gamma}\right\rangle=2 \rho_{\varsigma}^{2}-\rho\left(\rho_{\varsigma \varsigma}-\rho\right) / \sqrt{\rho^{2}+\rho_{\varsigma}^{2}}=0$, 
iff $\rho \rho_{\varsigma \varsigma}-2 \rho_{\varsigma}^{2}-\rho^{2}=0$ which means that $\varsigma$-parameter curves are asymptotic curves.

Corollary 11. Let $M^{*}$ be harmonic evolute surface (27) of tubular surface (12) via $\mathbb{B}$-Darboux frame in $E^{3}$. Then, the following are satisfying.

(1) $\mathbb{M}^{*}$ 's @-parameter curves not possible geodesic curves

(2) $\mathbb{M}^{*}$ 's $\varsigma$-parameter curves are geodesic curves iff

$$
\rho=c_{1} \cos \varsigma+c_{2} \sin \varsigma
$$

for any real constants $c_{1}$ and $c_{2}$.

Proof. If the acceleration vector of the parameter curve on the surface is parallel to the normal vector of the surface, the parameter curve is termed a geodesic curve. If that is the case, using (30) and (32), we have

$$
\text { (1) } \begin{aligned}
& \mathbb{Q}_{\Gamma} \times \Gamma_{\rho \rho}=\zeta_{2}(1-\lambda \rho)^{2} T+\rho_{\rho} \zeta_{2}(1-\lambda \rho) \mathbb{B}_{1}+\left[\rho_{\rho} \rho_{\rho \rho}\right. \\
& \left.+(1-\lambda \rho)\left[\rho_{\rho}\left(\zeta_{1}+2 \lambda\right)+\lambda_{\rho} \rho\right]\right] \mathbb{B}_{2} / \sqrt{\rho_{\rho}^{2}+(1-\lambda \rho)^{2}} \\
& \neq 0
\end{aligned}
$$

which means that $\rho$-parameter curves are not geodesic curves.

(2) $\mathbb{Q}_{\Gamma} \times \Gamma_{\varsigma \varsigma}=-\left[2 \rho \rho_{\varsigma}+\rho_{\varsigma}\left(\rho_{\varsigma \varsigma}-\rho\right)\right] T / \sqrt{\rho^{2}+\rho_{\varsigma}^{2}}$

Then, $\mathbb{Q}_{\Gamma} \times \Gamma_{\varsigma \varsigma}=0$ if only and only if $\rho_{\zeta}\left(\rho_{\zeta \varsigma}+\rho\right)=0$. This implies that the $\varsigma$-parameter curves are geodesic curves if the differential equation $\rho_{\varsigma \varsigma}+\rho=0$ has a solution $\rho=c_{1} \cos \varsigma+c_{2} \sin \varsigma$.

\section{Example}

Let $\mu$ be a circular helix parameterized as $\mu(\rho)=(\cos (\rho)$ $\sqrt{2}), \sin (\rho / \sqrt{2}), \rho / \sqrt{2})$. Then, the curve's Darboux frame and curvatures $\kappa_{g}, \kappa_{\mathrm{n}}$, and $\tau_{g}$ along $\mu(\rho)$ are dictated by

$$
\begin{aligned}
T(\rho) & =\left(-\frac{1}{\sqrt{2}} \sin \left(\frac{\rho}{\sqrt{2}}\right), \frac{1}{\sqrt{2}} \cos \left(\frac{\rho}{\sqrt{2}}\right), \frac{1}{\sqrt{2}}\right), \\
P(\rho) & =\left(\frac{1}{\sqrt{2}} \cos \left(\frac{\rho}{\sqrt{2}}\right)-\frac{1}{2} \sin \left(\frac{\rho}{\sqrt{2}}\right), \frac{1}{\sqrt{2}} \sin \left(\frac{\rho}{\sqrt{2}}\right)+\frac{1}{2} \cos \left(\frac{\rho}{\sqrt{2}}\right),-\frac{1}{2}\right), \\
\mathbb{Q}(\rho) & =\left(\frac{1}{\sqrt{2}} \cos \left(\frac{\rho}{\sqrt{2}}\right)+\frac{1}{2} \sin \left(\frac{\rho}{\sqrt{2}}\right), \frac{1}{\sqrt{2}} \sin \left(\frac{\rho}{\sqrt{2}}\right)-\frac{1}{2} \cos \left(\frac{\rho}{\sqrt{2}}\right), \frac{1}{2}\right), \\
\kappa_{g} & =-\frac{1}{2 \sqrt{2}}, \kappa_{n}=-\frac{1}{2 \sqrt{2}}, \tau_{g}=-\frac{1}{2} .
\end{aligned}
$$

Now, $\phi=\int_{0}^{\rho} \tau_{g} d t=\int_{0}^{\rho}-1 / 2 d t=-\rho / 2$. So, the $\mathbb{B}$-Darboux curvatures are calculated as

$$
\begin{aligned}
& \zeta_{1}=\frac{1}{2 \sqrt{2}}\left[\sin \left(\frac{\rho}{2}\right)-\cos \left(\frac{\rho}{2}\right)\right], \\
& \zeta_{2}=\frac{1}{2 \sqrt{2}}\left[\sin \left(\frac{\rho}{2}\right)+\cos \left(\frac{\rho}{2}\right)\right] .
\end{aligned}
$$

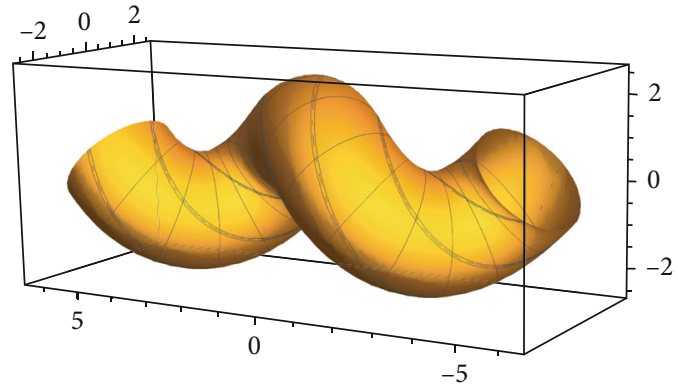

Figure 1: Tubular surface $\Omega_{1}$ due to Darboux frame.

Then, the $\mathbb{B}$-Darboux frame are given as

$$
\begin{aligned}
& T(\rho)=\frac{1}{\sqrt{2}}\left(-\sin \left(\frac{\rho}{\sqrt{2}}\right), \cos \left(\frac{\rho}{\sqrt{2}}\right), 1\right) \\
& \mathbb{B}_{1}(\rho)=\frac{1}{\sqrt{2}}\left[\cos \left(\frac{\rho}{\sqrt{2}}\right)\left[\cos \left(\frac{\rho}{2}\right)-\sin \left(\frac{\rho}{2}\right)\right]\right. \\
&+\frac{1}{\sqrt{2}} \sin \left(\frac{\rho}{\sqrt{2}}\right)\left[\cos \left(\frac{\rho}{2}\right)+\sin \left(\frac{\rho}{2}\right)\right], \sin \left(\frac{\rho}{\sqrt{2}}\right) \\
& \cdot\left[\cos \left(\frac{\rho}{2}\right)-\sin \left(\frac{\rho}{2}\right)\right]-\frac{1}{\sqrt{2}} \cos \left(\frac{\rho}{\sqrt{2}}\right) \\
&\left.\cdot\left[\cos \left(\frac{\rho}{2}\right)+\sin \left(\frac{\rho}{2}\right)\right], \frac{1}{\sqrt{2}}\left[\cos \left(\frac{\rho}{2}\right)+\sin \left(\frac{\rho}{2}\right)\right]\right] \\
& \mathbb{B}_{2}(\rho)=\frac{1}{\sqrt{2}}\left[\cos \left(\frac{\rho}{\sqrt{2}}\right)\left[\cos \left(\frac{\rho}{2}\right)+\sin \left(\frac{\rho}{2}\right)\right]\right. \\
&-\frac{1}{\sqrt{2}} \sin \left(\frac{\rho}{\sqrt{2}}\right)\left[\cos \left(\frac{\rho}{2}\right)-\sin \left(\frac{\rho}{2}\right)\right], \sin \left(\frac{\rho}{\sqrt{2}}\right) \\
& \cdot\left[\cos \left(\frac{\rho}{2}\right)+\sin \left(\frac{\rho}{2}\right)\right]-\frac{1}{\sqrt{2}} \cos \left(\frac{\rho}{\sqrt{2}}\right) \\
&\left.\cdot\left[\cos \left(\frac{\rho}{2}\right)-\sin \left(\frac{\rho}{2}\right)\right],-\frac{1}{\sqrt{2}}\left[\cos \left(\frac{\rho}{2}\right)-\sin \left(\frac{\rho}{2}\right)\right]\right]
\end{aligned}
$$

As a result and taking $r=\sqrt{2}$, the parameterization of the tubular surface $\mathbb{M}_{1}$ over the curve $\mu$ can be compiled in Darboux frame as $\Omega_{1}(\rho, \varsigma)=\mu(\rho)+r[\cos \varsigma \mathrm{P}(\rho)+\sin \varsigma \mathbb{Q}(\rho)]$ (see Figure 1), then we have

$$
\begin{aligned}
\Omega_{1}(\rho, \varsigma)= & {\left[\cos \left(\frac{\rho}{\sqrt{2}}\right)[1-\cos \varsigma+\sin \varsigma]+\frac{1}{\sqrt{2}} \sin \right.} \\
& \left.\cdot\left(\frac{\rho}{\sqrt{2}}\right)[\cos \varsigma+\sin \varsigma]\right], \sin \left(\frac{\rho}{\sqrt{2}}\right)[1-\cos \varsigma+\sin \varsigma] \\
& \left.-\frac{1}{\sqrt{2}} \cos \left(\frac{\rho}{\sqrt{2}}\right)[\cos \varsigma+\sin \varsigma], \frac{1}{\sqrt{2}}[\rho+\cos \varsigma+\sin \varsigma]\right] .
\end{aligned}
$$

The harmonic surface $\Gamma_{1}$ of $\Omega_{1}$ via Darboux frame $\Gamma_{1}(\rho, \varsigma)=\Omega_{1}(\rho, \varsigma)+\left(1 /\left(H_{\Omega_{1}}(\rho, \varsigma)\right)\right) \mathbb{Q}(\rho, \varsigma)$ can be given as (see Figure 2) 


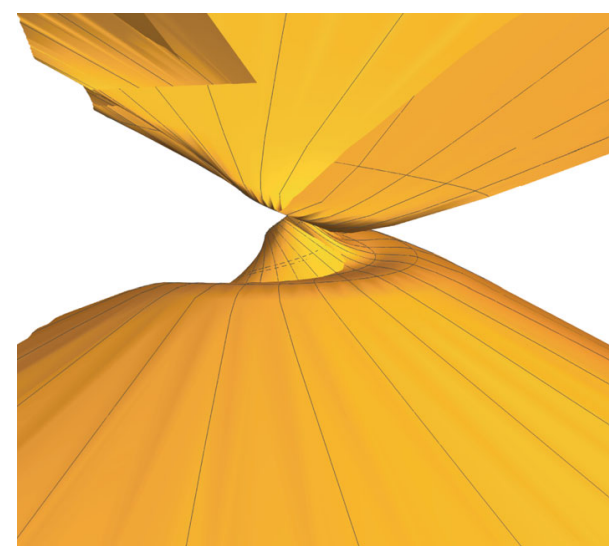

Figure 2: Harmonic surface $\Gamma_{1}$ via Darboux frame.

$$
\begin{aligned}
\Gamma_{1}(\rho, \varsigma)= & (1+\cos \varsigma+\sin \varsigma) \cos \left(\frac{\rho}{\sqrt{2}}\right)+\left(\frac{2-\cos \varsigma-\sin \varsigma}{1-\cos \varsigma-\sin \varsigma}\right) \\
& \cdot\left[\cos \left(\frac{\rho}{\sqrt{2}}\right)-\frac{1}{\sqrt{2}} \sin \left(\frac{\rho}{\sqrt{2}}\right)\right] \\
& -\frac{1}{\sqrt{2}} \sin \left(\frac{\rho}{\sqrt{2}}\right)[\cos \varsigma-\sin \varsigma],(1+\cos \varsigma+\sin \varsigma) \sin \\
& \cdot\left(\frac{\rho}{\sqrt{2}}\right)+\left(\frac{2-\cos \varsigma-\sin \varsigma}{1-\cos \varsigma-\sin \varsigma}\right)\left[\sin \left(\frac{\rho}{\sqrt{2}}\right)-\frac{1}{\sqrt{2}} \cos \left(\frac{\rho}{\sqrt{2}}\right)\right] \\
& +\frac{1}{\sqrt{2}} \cos \left(\frac{\rho}{\sqrt{2}}\right)[\cos \varsigma-\sin \varsigma], \frac{\rho}{2}-\frac{1}{\sqrt{2}}[\cos \varsigma-\sin \varsigma] \\
& \left.+\frac{2-\cos \varsigma-\sin \varsigma}{\sqrt{2}(1-\cos \varsigma-\sin \varsigma)}\right\} .
\end{aligned}
$$

From (12), the tubular surface $\Omega_{2}$ over the curve $\mu$ via $\mathbb{B}$ -Darboux frame can be given as (see Figure 3)

$$
\begin{aligned}
\Omega_{2}(\rho, \varsigma)= & {\left[\cos \left(\frac{\rho}{\sqrt{2}}\right)+\left[\cos \left(\frac{\rho}{2}\right)+\sin \left(\frac{\rho}{2}\right)\right]\right.} \\
& \cdot\left[\frac{1}{\sqrt{2}} \cos \varsigma \sin \left(\frac{\rho}{\sqrt{2}}\right)+\sin \varsigma \cos \left(\frac{\rho}{\sqrt{2}}\right)\right] \\
& +\left[\cos \left(\frac{\rho}{2}\right)-\sin \left(\frac{\rho}{2}\right)\right] \\
& \cdot\left[\cos \varsigma \cos \left(\frac{\rho}{\sqrt{2}}\right)-\frac{1}{\sqrt{2}} \sin \varsigma \sin \left(\frac{\rho}{\sqrt{2}}\right)\right], \sin \left(\frac{\rho}{\sqrt{2}}\right) \\
& +\left[\cos \left(\frac{\rho}{2}\right)+\sin \left(\frac{\rho}{2}\right)\right]\left[\sin \varsigma \sin \left(\frac{\rho}{\sqrt{2}}\right)\right. \\
& \left.-\frac{1}{\sqrt{2}} \cos \varsigma \cos \left(\frac{\rho}{\sqrt{2}}\right)\right]+\left[\cos \left(\frac{\rho}{2}\right)-\sin \left(\frac{\rho}{2}\right)\right] \\
& \cdot\left[\cos \varsigma \sin \left(\frac{\rho}{\sqrt{2}}\right)-\frac{1}{\sqrt{2}} \sin \varsigma \cos \left(\frac{\rho}{\sqrt{2}}\right)\right], \frac{1}{\sqrt{2}} \\
& \left.\cdot\left[\rho+\cos \left(\varsigma-\frac{\rho}{2}\right)-\sin \left(\varsigma-\frac{\rho}{2}\right)\right]\right] .
\end{aligned}
$$

Using (27), then the harmonic surface $\Gamma_{2}$ of $\Omega_{2}$ via $\mathbb{B}$-Darboux frame can be given as (see Figure 4)

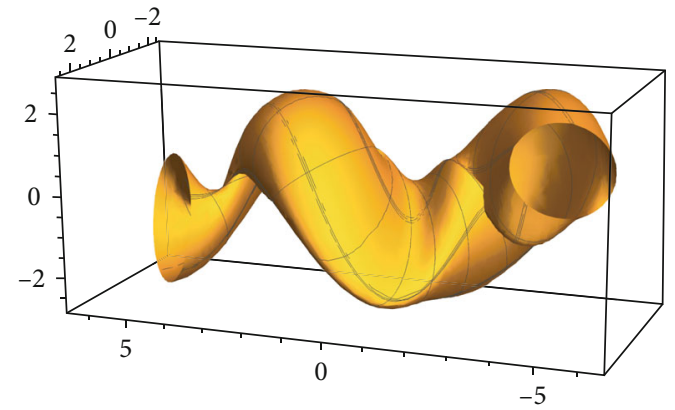

Figure 3: Tubular surface $\Omega_{2}$ via $\mathbb{B}$-Darboux frame.

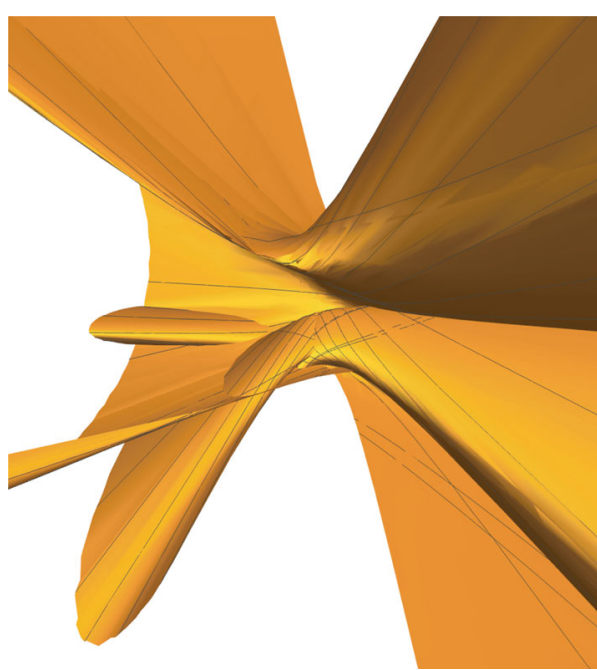

Figure 4: Harmonic surface $\Gamma_{2}$ via $\mathbb{B}$-Darboux frame.

$$
\begin{aligned}
\Gamma_{2}(\rho, \varsigma)= & \left\{\cos \left(\frac{\rho}{\sqrt{2}}\right)-\frac{1}{1+\cos (\varsigma+(\rho / 2))-\sin (\varsigma+(\rho / 2))}\right. \\
& \cdot\left[[ \operatorname { c o s } ( \frac { \rho } { 2 } ) - \operatorname { s i n } ( \frac { \rho } { 2 } ) ] \left[\cos \varsigma \cos \left(\frac{\rho}{\sqrt{2}}\right)\right.\right. \\
& \left.-\frac{1}{\sqrt{2}} \sin \varsigma \sin \left(\frac{\rho}{\sqrt{2}}\right)\right]+\left[\cos \left(\frac{\rho}{2}\right)+\sin \left(\frac{\rho}{2}\right)\right] \\
& \left.\cdot\left[\sin \varsigma \cos \left(\frac{\rho}{\sqrt{2}}\right)+\frac{1}{\sqrt{2}} \cos \varsigma \sin \left(\frac{\rho}{\sqrt{2}}\right)\right]\right], \sin \left(\frac{\rho}{\sqrt{2}}\right) \\
& -\frac{1}{1+\cos (\varsigma+(\rho / 2))-\sin (\varsigma+(\rho / 2))} \\
& \cdot\left[[ \operatorname { c o s } ( \frac { \rho } { 2 } ) - \operatorname { s i n } ( \frac { \rho } { 2 } ) ] \left[\cos \varsigma \sin \left(\frac{\rho}{\sqrt{2}}\right)\right.\right. \\
& \left.-\frac{1}{\sqrt{2}} \sin \varsigma \cos \left(\frac{\rho}{\sqrt{2}}\right)\right]-\left[\cos \left(\frac{\rho}{2}\right)+\sin \left(\frac{\rho}{2}\right)\right] \\
& \left.\cdot\left[\cos \varsigma \cos \left(\frac{\rho}{\sqrt{2}}\right)-\frac{1}{\sqrt{2}} \sin \varsigma \sin \left(\frac{\rho}{\sqrt{2}}\right)\right]\right], \frac{\rho}{\sqrt{2}} \\
& \left.-\frac{\sqrt{2}[\cos (\varsigma-(\rho / 2))-\sin (\varsigma-(\rho / 2))]}{1+\cos (\varsigma+(\rho / 2))-\sin (\varsigma+(\rho / 2))}\right\}
\end{aligned}
$$

\section{Conclusion}

Many researchers have recently researched curves and surfaces using the Bishop frame, similar to how they studied 
curves and surfaces using the Frenet frame. The concept of a $\mathbb{B}$-Darboux frame was recently shown, and there is a chance that the further studies may be conducted in the future. We study the characterisation of tubular surfaces using the $\mathbb{B}$-Darboux frame and the harmonic surface of tubular surfaces using the $\mathbb{B}$-Darboux frame in this paper. We provide the required and sufficient circumstances for a tubular surface to become a developable and minimum surface using the $\mathbb{B}$-Darboux frame. Furthermore, they demonstrate that the harmonic surface of a tubular surface is neither a developable nor a minimal surface.

\section{Data Availability}

No data is used in this study.

\section{Conflicts of Interest}

The authors declare no competing interest.

\section{Authors' Contributions}

All authors have equal contribution and finalized the paper.

\section{Acknowledgments}

The authors extend their appreciation to the Deanship of Scientific Research at Imam Mohammad Ibn Saud Islamic University for funding this work through research group no. RG-21-09-04.

\section{References}

[1] M. Dede, M. C. Aslan, and C. Ekici, "On a variational problem due to the B-Darboux frame in Euclidean 3-space," Mathematical Methods in the Applied Sciences, vol. 44, pp. 1-9, 2021.

[2] E. M. Solouma, "Investigation of non-lightlike tubular surfaces with Darboux frame in Minkowski 3-space," Nonlinear Analysis and Differential Equations, vol. 4, no. 10, pp. 493-502, 2016.

[3] L. R. Bishop, "There is more than one way to frame a curve," The American Mathematical Monthly, vol. 82, no. 3, pp. 246-251, 1975.

[4] K. Eren, "On the harmonic evolute surfaces of tubular surfaces in Euclidean 3-space," Journal of Science and Arts, vol. 21, no. 2, pp. 449-460, 2021.

[5] K. Eren and A. K. Akbay, "On the harmonic evolute surfaces of Hasimoto surfaces," Adryaman University Journal of Science, vol. 11, no. 1, pp. 87-100, 2021.

[6] T. Körpinar and G. U. Kaymanli, "On the harmonic evolute of quasi normal surfaces," Journal of Science and Arts, vol. 1, no. 50, pp. 55-64, 2020.

[7] R. Lopez, Z. M. Sipus, L. P. Gajcic, and I. Protrka, "Harmonic evolutes of B-scrolls with constant mean curvature in LorentzMinkowski space," International Journal of Geometric Methods in Modern Physics, vol. 16, no. 5, article 1950076, 2019.

[8] D. J. Struik, Lectures on Classical Differential Geometry, Dover, Addison Wesley, 2nd edition, 1988.

[9] J. L. M. Barbosa and A. G. Colares, Minimal Surfaces in $R^{3}$, Springer Verlag, Berlin Heidelberg, 1986.
[10] M. P. Do Carmo, Differential Geometry of Curves and Surfaces: Revised and Updated Second Edition, Courier Dover Publications, 2016.

[11] G. Hu, H. Cao, J. Wu, and G. Wei, "Construction of developable surfaces using generalized C-Bézier bases with shape parameters," Computational and Applied Mathematics, vol. 39, no. 3, 2020.

[12] S. Hu, Z. Wang, and X. Tang, "Tubular surfaces of center curves on spacelike surfaces in Lorentz-Minkowski 3-space," Mathematical Methods in the Applied Sciences, vol. 42, no. 9, pp. 3136-3166, 2019.

[13] J. Qian, J. Liu, X. Fu, and S. D. Jung, "Geometric characterizations of canal surfaces with Frenet center curves," AIMS Mathematics, vol. 6, no. 9, pp. 9476-9490, 2021. 